\title{
Efeito da Cobertura Morta de Milheto (Pennisetum americanum) SOBRe A EFICÁCIA do Herbicida Metribuzin No ConTROle de Ipomoea grandifolia $\mathrm{E}$ Sida rhombifolia
}

\author{
Effect of Pennisetum americanum Mulch in the Metribuzin Efficacy on the Control of \\ Ipomoea grandifolia and Sida rhombifolia
}

\begin{abstract}
GODOY, M.C. ${ }^{2}$, MESCHEDE, D.K. ${ }^{3}$, CARBONARI, C.A. ${ }^{4}$, CORREIA, M.R. ${ }^{5}$ e VELINI, E.D. ${ }^{6}$
RESUMO - Com o objetivo de avaliar a eficácia do metribuzin (480 g i.a. ha ${ }^{-1}$ ) associado à palha de milheto no controle de Ipomoea grandifolia e Sida rhombifolia, foram realizados dois experimentos em casa de vegetação. No primeiro, os tratamentos constituíram-se de diferentes posicionamentos do herbicida, aplicado sobre e sob a palha em diferentes condições de umidade. No segundo, foram estudados diferentes períodos de permanência $(0,7,14$ e 21 dias) do herbicida sobre a palha de milheto antes da ocorrência da primeira chuva. Após o preenchimento dos vasos com solo, as plantas daninhas (I. grandifolia e S. rhombifolia) foram semeadas superficialmente e, em seguida, cobertas com palha de milheto $\left(8 \mathrm{t} \mathrm{ha}^{-1}\right)$. O delineamento experimental utilizado em ambos os experimentos foi o inteiramente casualizado, com quatro repetições. Foram realizadas avaliações visuais de controle (0 a 100\%), contagem das plantas daninhas aos 7, 14, 21, 28 e 35 dias após a aplicação (DAA) e biomassa seca ao final. Verificouse controle excelente das duas espécies nos diferentes posicionamentos do herbicida, exceto para I. grandifolia na condição de aplicação em palha úmida, seguido de período seco. Observouse, ainda, que o herbicida promoveu controle eficaz em pós-emergência e em pré-emergência, mesmo sem ocorrência de chuva após a aplicação. No segundo experimento, constatou-se controle excelente $(>96 \%)$ de I. grandifolia nos os períodos sem chuva de até 7 DAA; nos demais periodos, tal controle foi insatisfatório. Para S. rhombifolia, observou-se controle excelente para os períodos até 14 dias sem ocorrência de chuvas. Para o período de 28 dias, não se obteve controle satisfatório.
\end{abstract}

Palavras-chave: planta daninha, cobertura morta, milheto, metribuzin.

\begin{abstract}
Two experiments were carried out under greenhouse conditions to evaluate the efficacy of the metribuzin herbicide associated to Pennisetum americanum mulch in the control of the weeds Ipomoea grandifolia and Sida rhombifolia. The treatments in the first experiment were constituted by different metribuzin application positions, over and under the straw, under different humidity conditions. In the second experiment, different periods of metribuzin permanence on the mulch before the occurrence of the first rain were observed on 0, 7, 14, 21 and 28 days after herbicide application on the mulch. The vases were filled up with soil and the weeds were sowed superficially and covered with $\boldsymbol{P}$. americanum (8 $\left.t \mathrm{ha}^{-1}\right)$ mulch. The experimental design was randomized with four repetitions. The metribuzin dose used was $480 \mathrm{~g} \mathrm{ha}^{-1}$ (a.i.). The control was evaluated visually, by percentile note scale and by counting the plants and evaluating plant dry mass. In the first experiment, an excellent control of the two species studied in the different positions of the tested herbicide was observed, except for I. grandifolia applied on the humidity mulch. The herbicide promoted an efficient control in post-emergence and pre-emergence, even when absorbed by direct contact with the mulch. In
\end{abstract}

Recebido para publicação em 7.6.2006 e na forma revisada em 27.2.2007.

2 Eng.-Agr. aluna de pós-graduação, nível mestrado, Faculdade de Ciências Agrárias, Universidade Estadual Paulista FCA/UNESP, Fazenda Experimental Lageado, Caixa Postal 237, 18603-970 Botucatu-SP; ${ }^{3}$ Eng.-Agr. aluna de pós-graduação, nível doutorado, FCA/UNESP-B otucatu-SP; ${ }^{4}$ Eng.-Agr. aluno de pós-graduação, nível mestrado, FCA/UNESP-B otucatu-SP; ${ }^{5}$ Eng.-Agr. Dr., FCA/UNESP-B otucatu-SP; ${ }^{6}$ Prof. Dr., Dep. Agricultura - FCA/ UNESP-Botucatu-SP. 
the second experiment, an excellent I. grandifolia control was observed in the periods without rain until 7 days after application (>96\%), being unsatisfactory in the other periods. S. rhombifolia showed an excellent control during the periods up to 14 days without rain, presenting a satisfactory control even up to a 21 day period. The 28-day period did not present a satisfactory control.

Keywords: weed, mulch, Pennisetum americanum, metribuzin.

\section{INTRODUÇÃO}

Nos últimos anos, tem-se observado, no Brasil, rápida expansão de áreas com culturas implantadas sobre algum tipo de cobertura morta. Entre exemplos típicos, pode-se mencionar o cultivo mínimo em áreas de reflorestamento, com manutenção da serrapilheira sobre o solo, o sistema de produção de cana-crua e, com maior adoção, o plantio direto de culturas anuais. O milheto (Pennisetum americanum) produz boa quantidade de biomassa e, por isso, viabilizou o estabelecimento do sistema de semeadura direta no cerrado, além de servir para rotação e sucessão a outras culturas.

Vários trabalhos destacam a importância da manutenção das coberturas mortas sobre a superficie do solo, sendo uma das características o melhor manejo das plantas daninhas. Alguns estudos têm comprovado a eficácia da cobertura morta na redução da população de plantas daninhas (Azania et al., 2002; Lorenzi, 1984, 1993; Correia \& Durigan, 2004; Martins et al., 1999). No entanto, nesses sistemas de produção podem ocorrer grandes variações na quantidade e na composição da cobertura morta, que pode ou não suprimir a germinação da comunidade infestante, bem como influenciar a dinâmica de herbicidas aplicados sobre a palhada, em ocasiões em que o controle se faz necessário.

Há controvérsia em relação à influência da cobertura morta na eficácia dos herbicidas aplicados em pré-emergência. Enquanto alguns defendem a hipótese de que em sistema de plantio direto pode-se reduzir ou até mesmo eliminar a aplicação de herbicida em pré-emergência, pelo efeito físico e/ou alelopático das coberturas (Velini \& Negrisoli, 2000), outros defendem a necessidade de aumento da dose, pelo fato de que parte do produto ficaria retida na palha e não conseguiria atingir o solo.
Estudos realizados por Pastana (1972), citado por Rodrigues et al. (1998), mostraram que, quando atrazine foi aplicado em solo com cobertura morta de aveia, o peso seco das plantas daninhas que ali se desenvolveram foi maior que em solo sem cobertura morta, sugerindo que parte do produto infestante ficou retida na palha. A redução do peso seco das infestantes, em solo com cobertura, foi conseguida com o aumento da dose do produto.

Os herbicidas residuais, para exercerem sua atividade sobre as plantas daninhas, precisam ser introduzidos diretamente no solo, o que se dá através da incorporação pela chuva, e depende da solubilidade do produto (Rossi, 2004). Nesse sentido, a manutenção das coberturas mortas sobre a superficie do solo pode, simultaneamente, reduzir o potencial de infestação da comunidade infestante, como também dificultar o desempenho de herbicidas, uma vez que os principais componentes da dinâmica de herbicidas na palha são a transposição do produto através desta e a própria dinâmica de molhamento e lavagem da palha pela água das chuvas (Maciel \& Velini, 2005).

A capacidade de um herbicida residual atingir o solo, no sistema de plantio direto, não depende apenas da solubilidade em água e da volatilidade do produto, mas também da quantidade e origem da cobertura morta, da quantidade e época da primeira irrigação após a aplicação do produto (e das irrigações subseqüentes) e das condições climáticas prevalecentes durante e após aplicação (Maciel, 2001).

O metribuzin é um herbicida sistêmico seletivo, do grupo das triazinonas, usado em pré e pós-emergência no controle de muitas gramineas e plantas daninhas de folha larga em diversas culturas; é altamente solúvel em água $\left(1,05 \mathrm{~g} \mathrm{~L}^{-1}\right)$ e seu coeficiente de sorção varia de 0,56 em solos areno-argilosos a 31,7 
em solos com $60 \%$ de matéria orgânica. Sua persistência nos solos é considerada pouca a moderada, e a meia-vida, mensurada entre 5 e 50 dias (Huertas-Pérez, 2005). A grande perda de metribuzin nos solos tem sido atribuída mais a processos de degradação que a processos de transferência, Savage (1980) demonstrou que 10 a $12 \%$ do metribuzin aplicado na superficie do solo foi perdido por volatilização. Banks \& Robinson, em 1982, estudaram a influência da palha do trigo no comportamento do metribuzin no solo e verificaram que menos de $1 \%$ desse herbicida alcançou o solo antes da irrigação. Por outro lado, quando as parcelas foram irrigadas logo após a aplicação, observou-se que $56 \%$ do metribuzin atingiu o solo.

O objetivo deste trabalho foi avaliar a eficácia do metribuzin, aplicado à palha de milheto em diferentes posicionamentos e intervalos de chuva, no controle de Ipomoea grandifolia e Sida rhombifolia.

\section{MATERIAL E MÉTODOS}

Foram realizados dois experimentos em casa de vegetação no Núcleo de Pesquisas Avançadas em Matologia - NUPAM, pertencente ao Departamento de Produção Vegetal da Faculdade de Ciências Agronômicas/UNESP, campus de Botucatu/SP.

Foram utilizados vasos de 3 litros preenchidos com solo do tipo Latossolo Vermelho Distrófico (LVd), com pH de 4,3, teor de matéria orgânica em torno de $9,0 \mathrm{~g} \mathrm{dm}^{-3} \mathrm{e} \mathrm{V \%}$ de 21 ; em função dessa análise é que se fez a adubação.

No primeiro experimento, os tratamentos constituíram-se de diferentes posicionamentos do herbicida metribuzin associado à palha de milheto: 1 - aplicação de metribuzin sobre a palha seguida de simulação de chuva (30 $\mathrm{mm})$; 2 - simulação de chuva $(30 \mathrm{~mm})$ seguida da cobertura do solo com palha e aplicação de metribuzin na palha seca; 3 - simulação de chuva $(30 \mathrm{~mm})$ sobre a palha e aplicação de metribuzin em pós-emergência (plantas com duas a três folhas definitivas); 4 - simulação de chuva $(30 \mathrm{~mm})$ seguida da aplicação de metribuzin no solo e cobertura deste com palha seca; 5 - aplicação de metribuzin em palha seca e posterior simulação de chuva $(2,5 \mathrm{~mm}) ; 6$ aplicação de metribuzin sobre a palha seca e com irrigação dos vasos feita subsuperficialmente; 7 - aplicação de metribuzin em vasos sem palha e com irrigação feita subsuperficialmente; 8 - simulação de chuva $(30 \mathrm{~mm})$ sobre a palha e aplicação de metribuzin sobre palha úmida; 9 - testemunha com palha e sem aplicação; e 10 - testemunha sem palha e sem aplicação.

No segundo experimento, diferentes períodos de permanência do herbicida metribuzin sobre a palha de milheto, antes da ocorrência da primeira chuva $(30 \mathrm{~mm})$, foram testados: 0 , $7,14,21$ e 28 dias após aplicação do herbicida sobre a palha. As plantas daninhas estudadas foram Ipomoea grandifolia e Sida rhombifolia, as quais foram semeadas e incorporadas superficialmente ao solo (até $2 \mathrm{~cm}$ ); em seguida, os vasos foram cobertos com a palha de milheto em uma quantidade equivalente a $8 \mathrm{t} \mathrm{ha}^{-1}$, a qual foi previamente coletada em campo, picada em fragmentos de aproximadamente $5 \mathrm{~cm}$ e colocada para secar em estufa a $60^{\circ} \mathrm{C}$ por 72 horas.

Por ocasião da aplicação, a temperatura do ar foi de $23{ }^{\circ} \mathrm{C}$ e a umidade relativa de $35 \%$. As avaliações ocorreram no periodo de 7 a 35 dias após a simulação da chuva e foi composta pela análise da densidade de plantas por vaso e notas de controle, que seguiram escala de notas de 1 a $100 \%$ de controle.

A aplicação do herbicida e a simulação de chuva, em ambos os ensaios, foram realizadas através de um equipamento instalado em laboratório, constituído de duas barras de pulverização, uma responsável pelo sistema de simulação de chuva e a outra pelo sistema de pulverização de defensivos agricolas, as quais se deslocam por uma área útil de $6 \mathrm{~m}^{2}$ no sentido do comprimento do equipamento. A aplicação do herbicida foi feita com gasto de calda correspondente a $200 \mathrm{~L} \mathrm{ha}^{-1}$, e a dose deste foi de $480 \mathrm{~g} \mathrm{ha}^{-1}$ de i.a. O equipamento consistiu de uma barra com quatro pontas do tipo XR1 1002, sendo operado sob pressão constante de 1,5 bar, pressurizado por ar comprimido.

Para simulação, a lâmina aplicada correspondeu a aproximadamente $2,5 \mathrm{~mm}$ de chuva.

Após a aplicação dos tratamentos, os vasos foram irrigados com auxílio de pisetas, de forma que a palha não fosse lavada. As avaliações 
foram feitas semanalmente, no periodo de 7 a 35 dias após a aplicação, no primeiro experimento, e após a ocorrência de chuvas, no segundo experimento. A avaliação constituiu-se da contagem da densidade de plantas em cada vaso e da avaliação visual de controle através de uma escala percentual de notas, em que 0 (zero) correspondeu à ausência de controle e 100 ao controle total das plantas. No encerramento dos experimentos, aos 35 DAA, foi realizada avaliação da biomassa seca das plantas. Para isso, estas foram cortadas e colocadas para secar em estufa de circulação forçada de ar a $60^{\circ} \mathrm{C}$ por 72 horas.

Os tratamentos foram dispostos em um delineamento experimental inteiramente casualizado, com quatro repetições. Os dados foram avaliados por meio da análise de variância pelo teste $\mathrm{F}$, sendo as médias dos tratamentos comparadas pelo teste $t$ a $5 \%$ de probabilidade.

\section{RESULTADOS E DISCUSSÃO}

Pode-se observar, aos 7 DAA, que os tratamentos em que o produto foi aplicado diretamente sobre o solo, seguido ou não da cobertura com palha, e no qual ele foi aplicado sobre a palha, recebendo em seguida chuva de $2,5 \mathrm{~mm}$, proporcionaram melhor eficácia de controle: em torno de 60\% (Tabela 1). Banks (1982), trabalhando em uma condição de $9 \mathrm{t} \mathrm{ha}^{-1}$ de palha e $2,5 \mathrm{~mm}$ de chuva, observou que mais de $40 \%$ do metribuzin aplicado sobre a palha de trigo foi liberado para o solo. A partir dos 14 DAA, observou-se que o metribuzin promoveu elevados niveis de controle de I. grandifolia quando aplicado nas diferentes modalidades, com exceção do tratamento em que o produto foi aplicado sobre a palha úmida - neste caso, possivelmente devido à maior adsorção e indisponibilidade do produto. Vale ressaltar que o metribuzin promoveu controle excelente $(99,7 \%)$ mesmo quando aplicado na palha seca e sem ocorrência de chuvas, o que demonstra que ele pode ser absorvido diretamente pelo contato da planta daninha com a palha. Resultados semelhantes foram observados por Rossi (2004), que verificaram que o herbicida metribuzin foi absorvido diretamente da palha de cana-deaçúcar pelas espécies Euphorbia heterophylla, Brachiaria plantaginea e I. grandifolia.
Os resultados de densidade de plantas (Tabela 2) também demonstram o excelente controle de I. grandifolia proporcionado pelos diferentes posicionamentos do metribuzin, com exceção do tratamento com aplicação sobre palha úmida, que apresentou maior densidade de plantas quando comparado com os demais tratamentos com o herbicida. Pode-se observar, ainda, que a testemunha com palha não reduziu significativamente a densidade de plantas para esta espécie, porém promoveu redução de biomassa seca de aproximadamente 35\% (Tabela 2).

Para S. rhombifolia, verificou-se, aos 7 DAA, que os tratamentos em que o produto foi aplicado diretamente sobre o solo, seguido ou não da cobertura com palha, e tratamentos em que o herbicida foi aplicado sobre a palha, recebendo em seguida chuva $(2,5 \mathrm{~mm})$, apresentaram os melhores resultados de controle (Tabela 3). A partir dos 21 DAA, observou-se que o metribuzin promoveu controle excelente de $S$. rhombifolia quando aplicado nos diferentes posicionamentos, mesmo quando aplicado sobre a palha úmida. Observou-se, ainda, eficácia de controle excelente das plantas pelo contato direto do herbicida na palha, sem ocorrência de chuvas após a aplicação. Esses resultados também foram observados para densidade e biomassa seca das plantas de S. rhombifolia (Tabela 4). Para este herbicida, a presença da palha não reduziu sua densidade em relação à testemunha sem palha, mas promoveu redução de biomassa seca em $31,5 \%$.

Na Tabela 5 estão apresentados os resultados de controle de I. grandifolia para o estudo de periodos de permanência do produto na palha antes da ocorrência da primeira chuva. Foram constatados resultados satisfatórios de controle de plantas daninhas quando ocorreram chuvas até 14 dias após a aplicação do herbicida sobre a palha, permitindo a germinação das plantas daninhas e carregando o produto para o solo. No entanto, para biomassa seca, observou-se redução significativa nos períodos sem ocorrência de chuvas de até 21 dias (Tabela 6). Maciel (2001) e Tofoli (2004) ressaltam a importância da época de ocorrência da primeira chuva após a aplicação do produto no carreamento do herbicida da palha para o solo e a eficácia de controle das plantas daninhas. 
Tabela 1 - Porcentagem de controle de Ipomoea grandifolia nos diferentes posicionamentos do herbicida metribuzin associado à palha de milheto

\begin{tabular}{|c|c|c|c|c|c|}
\hline \multirow{2}{*}{ Tratamento } & \multicolumn{5}{|c|}{ Porcentagem de controle } \\
\hline & 7 DAA & $14 \mathrm{DAA}$ & $21 \mathrm{DAA}$ & $28 \mathrm{DAA}$ & 35 DAA \\
\hline 1 - metribuzin sobre a palha - chuva $(30 \mathrm{~mm})$ & $12,5 \mathrm{BC}$ & $99,2 \mathrm{~A}$ & $99,0 \mathrm{~A}$ & $99,7 \mathrm{~A}$ & $99,7 \mathrm{~A}$ \\
\hline 2 - chuva $(30 \mathrm{~mm})$ sobre o solo - metribuzin sobre a palha seca & $41,5 \mathrm{BC}$ & $89,5 \mathrm{~A}$ & $99,5 \mathrm{~A}$ & $98,7 \mathrm{~A}$ & $99,7 \mathrm{~A}$ \\
\hline 3 - chuva $(30 \mathrm{~mm})$ - metribuzin sobre a palha seca em pós -emergência & $0,0 \mathrm{C}$ & $86,7 \mathrm{~A}$ & $74,5 \mathrm{AB}$ & $98,2 \mathrm{~A}$ & $98,7 \mathrm{~A}$ \\
\hline 4 - chuva $(30 \mathrm{~mm})$ no solo - metribuzin sob a palha seca & $62,7 \mathrm{~A}$ & $100,0 \mathrm{~A}$ & $100,0 \mathrm{~A}$ & $100,0 \mathrm{~A}$ & $100,0 \mathrm{~A}$ \\
\hline 5 - metribuzin sobre a palha seca - chuva de $2,5 \mathrm{~mm}$ & $55,0 \mathrm{~A}$ & $88,7 \mathrm{~A}$ & $99,5 \mathrm{~A}$ & $93,5 \mathrm{~A}$ & $99,0 \mathrm{~A}$ \\
\hline 6 - metribuzin sobre a palha seca - sem ocorrência de chuvas & $37,0 \mathrm{BC}$ & $92,7 \mathrm{~A}$ & $97,5 \mathrm{~A}$ & $96,0 \mathrm{~A}$ & $99,7 \mathrm{~A}$ \\
\hline 7 - metribuzin sem palha - sem ocorrência de chuvas & $65,0 \mathrm{~A}$ & $98,5 \mathrm{~A}$ & $99,5 \mathrm{~A}$ & $98,2 \mathrm{~A}$ & $100,0 \mathrm{~A}$ \\
\hline 8 - chuva $(30 \mathrm{~mm})$ sobre a palha - metribuzin & $7,5 \mathrm{BC}$ & $38,7 \mathrm{~B}$ & $58,0 \mathrm{~B}$ & $37,5 \mathrm{~B}$ & $47,5 \mathrm{~B}$ \\
\hline 9 - Testemunha com palha & $0,0 \mathrm{C}$ & $0,0 \mathrm{C}$ & $0,0 \mathrm{C}$ & $0,0 \mathrm{C}$ & $0,0 \mathrm{C}$ \\
\hline 10 - Testemunha sem palha & $0,0 \mathrm{C}$ & $0,0 \mathrm{C}$ & $0,0 \mathrm{C}$ & $0,0 \mathrm{C}$ & $0,0 \mathrm{C}$ \\
\hline F tratamento & $4,88^{* *}$ & $56,20^{* *}$ & $15,95^{* *}$ & $34,80 * *$ & $62,04 * *$ \\
\hline $\mathrm{CV}(\%)$ & 87,08 & 15,62 & 28,1 & 19,95 & 14,48 \\
\hline DMS & 35,4 & 15,7 & 29,5 & 20,8 & 15,6 \\
\hline
\end{tabular}

** significativo pelo teste $\mathrm{F}$ a $5 \%$ de probabilidade. Médias seguidas de mesma letra na coluna não diferem estatisticamente entre si pelo teste $t$ a $5 \%$ de probabilidade. DAA - dias após a aplicação do herbicida.

Tabela 2 - Densidade e biomassa seca das plantas de Ipomoea grandifolia nos diferentes posicionamentos do herbicida metribuzin associado à palha de milheto

\begin{tabular}{|c|c|c|c|c|c|c|}
\hline \multirow{2}{*}{ Tratamento } & \multicolumn{5}{|c|}{ Densidade de plantas por vaso } & \multirow{2}{*}{$\begin{array}{c}\text { Massa } \\
\text { seca } \\
(\mathrm{g})\end{array}$} \\
\hline & 7 DAA & $14 \mathrm{DAA}$ & $21 \mathrm{DAA}$ & 28 DAA & $35 \mathrm{DAA}$ & \\
\hline 1 - metribuzin sobre a palha - chuva $(30 \mathrm{~mm}) \quad$ & $15,7 \mathrm{BCD}$ & $0,7 \mathrm{D}$ & $0,2 \mathrm{D}$ & $0,0 \mathrm{C}$ & $0,0 \mathrm{C}$ & $0,00 \mathrm{D}$ \\
\hline 2 - chuva $(30 \mathrm{~mm})$ sobre o solo - metribuzin sobre a palha seca & $14,7 \mathrm{BCD}$ & $4,2 \mathrm{CD}$ & $1,2 \mathrm{D}$ & $1,2 \mathrm{C}$ & $0,2 \mathrm{C}$ & $0,00 \mathrm{D}$ \\
\hline 3 - chuva $(30 \mathrm{~mm})$ - metribuzin sobre a palha seca em pós-emergência & $0,0 \mathrm{E}$ & $10,7 \mathrm{C}$ & $0,7 \mathrm{D}$ & $0,7 \mathrm{C}$ & $0,7 \mathrm{C}$ & $0,00 \mathrm{D}$ \\
\hline 4 - chuva $(30 \mathrm{~mm})$ no solo - metribuzin sob a palha seca & $10,7 \mathrm{CD}$ & $0,2 \mathrm{D}$ & $0,0 \mathrm{D}$ & $0,0 \mathrm{C}$ & $0,0 \mathrm{C}$ & $0,00 \mathrm{D}$ \\
\hline 5 - metribuzin sobre a palha seca - chuva $(2,5 \mathrm{~mm})$ & $8,7 \mathrm{DE}$ & $8,0 \mathrm{C}$ & $1,0 \mathrm{D}$ & $0,0 \mathrm{C}$ & $0,5 \mathrm{C}$ & $0,00 \mathrm{D}$ \\
\hline 6 - metribuzin sobre a palha seca - sem ocorrência de chuvas & $13,0 \mathrm{CD}$ & $5,2 \mathrm{CD}$ & $0,2 \mathrm{D}$ & $0,0 \mathrm{C}$ & $0,2 \mathrm{C}$ & $0,00 \mathrm{D}$ \\
\hline 7 - metribuzin sem palha - sem ocorrência de chuvas & $17,5 \mathrm{BCD}$ & $1,2 \mathrm{D}$ & $0,2 \mathrm{D}$ & $0,2 \mathrm{C}$ & $0,0 \mathrm{C}$ & $0,00 \mathrm{D}$ \\
\hline 8 - chuva $(30 \mathrm{~mm})$ sobre a palha - metribuzin & $23,5 \mathrm{AB}$ & $10,7 \mathrm{C}$ & $7,7 \mathrm{C}$ & $7,2 \mathrm{~B}$ & $8,2 \mathrm{~B}$ & $1,50 \mathrm{C}$ \\
\hline 9 -Testemunha com palha & $20,2 \mathrm{BC}$ & $20,2 \mathrm{~B}$ & $22,7 \mathrm{~B}$ & $20,0 \mathrm{~A}$ & $20,0 \mathrm{~A}$ & $4,75 \mathrm{~B}$ \\
\hline 10 - Testemunha sem palha & $32,2 \mathrm{~A}$ & $32,2 \mathrm{~A}$ & $29,7 \mathrm{~A}$ & $23,7 \mathrm{~A}$ & $23,7 \mathrm{~A}$ & $7,3 \mathrm{~A}$ \\
\hline F tratamento & $6,09^{* * *}$ & $19,57 * *$ & $62,88 * *$ & $40,55^{* * *}$ & $42,99 * *$ & $27,67 * *$ \\
\hline $\mathrm{CV}(\%)$ & 45,22 & 48,60 & 42,70 & 53,31 & 51,60 & 62,54 \\
\hline DMS & 10,2 & 6,6 & 4,0 & 4,1 & 4,0 & 1,40 \\
\hline
\end{tabular}

** significativo pelo teste $\mathrm{F}$ a $5 \%$ de probabilidade. Médias seguidas de mesma letra na coluna não diferem estati sticamente entre si pelo teste $t$ a $5 \%$ de probabilidade. DAA - dias após a aplicação do herbicida.

Para S. rhombifolia, os resultados de controle observados com a ocorrência da primeira chuva até os 14 dias após a aplicação do herbicida foram satisfatórios, mostrando valores da ordem de $73,7 \%$ na avaliação feita 7 dias após a chuva e chegando a uma porcentagem de controle de 97,7\% no final da avaliação. Até 21 dias após a realização da primeira chuva, as porcentagens de controle ainda se mantiveram satisfatórias, porém somente na avaliação feita a partir dos 14 dias após a chuva (Tabela 7). Novamente, os resultados de densidade de plantas (Tabela 8) demonstraram boa eficácia de controle, com redução significativa das plantas nos períodos sem ocorrência de chuvas de até 21 dias; no entanto, para biomassa seca, observou-se redução significativa nos períodos sem ocorrência de chuvas de até 14 dias. 
Tabela 3 - Porcentagem de controle das plantas de Sida rhombifolia nos diferentes posicionamentos do herbicida metribuzin associado à palha de milheto

\begin{tabular}{|c|c|c|c|c|c|}
\hline \multirow{2}{*}{ Tratamento } & \multicolumn{5}{|c|}{ Porcentagem de controle } \\
\hline & 7 DAA & 14 DAA & 21 DAA & 28 DAA & 35 DAA \\
\hline 1 - metribuzin sobre a palha - chuva $(30 \mathrm{~mm})$ & $28,7 \mathrm{C}$ & $99,7 \mathrm{~A}$ & $97,2 \mathrm{~A}$ & $100,0 \mathrm{~A}$ & $100,0 \mathrm{~A}$ \\
\hline 2 - chuva $(30 \mathrm{~mm})$ sobre o solo - metribuzim sobre a palha seca & $17,5 \mathrm{C}$ & $97,7 \mathrm{~A}$ & $99,5 \mathrm{~A}$ & $99,5 \mathrm{~A}$ & $98,7 \mathrm{~A}$ \\
\hline 3 - chuva $(30 \mathrm{~mm})$ - metribuzin sobre a palha seca em pós-emergência & $0,0 \mathrm{D}$ & $80,0 \mathrm{C}$ & $25,0 \mathrm{~B}$ & $25,0 \mathrm{~B}$ & $100,0 \mathrm{~A}$ \\
\hline 4 - chuva $(30 \mathrm{~mm})$ no solo - metribuzin sobre a palha seca & $73,7 \mathrm{~A}$ & $98,7 \mathrm{~A}$ & $100,0 \mathrm{~A}$ & $100,0 \mathrm{~A}$ & $100,0 \mathrm{~A}$ \\
\hline 5 - metribuzin sobre a palha seca - chuva $(2,5 \mathrm{~mm})$ & $79,5 \mathrm{~A}$ & $87,5 \mathrm{~B}$ & $98,5 \mathrm{~A}$ & $100,0 \mathrm{~A}$ & $100,0 \mathrm{~A}$ \\
\hline 6 - metribuzin sobre a palha seca - sem ocorrência de chuvas & $58,7 \mathrm{~B}$ & $99,7 \mathrm{~A}$ & $99,2 \mathrm{~A}$ & $100,0 \mathrm{~A}$ & $100,0 \mathrm{~A}$ \\
\hline 7 - metribuzin sem palha - sem ocorrência de chuvas & $83,7 \mathrm{~A}$ & $89,2 \mathrm{~B}$ & $96,7 \mathrm{~A}$ & $99,0 \mathrm{~A}$ & $97,0 \mathrm{~B}$ \\
\hline 8 - chuva $(30 \mathrm{~mm})$ sobre a palha - metribuzin & $15,0 \mathrm{C}$ & $90,0 \mathrm{~B}$ & $98,0 \mathrm{~A}$ & $97,0 \mathrm{~A}$ & $99,0 \mathrm{~A}$ \\
\hline 9 - Testemunha com palha & $0,0 \mathrm{D}$ & $0,0 \mathrm{D}$ & $0,0 \mathrm{C}$ & $0,0 \mathrm{C}$ & $0,0 \mathrm{C}$ \\
\hline 10 - Testemunha sem palha & $0,0 \mathrm{D}$ & $0,0 \mathrm{D}$ & $0,0 \mathrm{C}$ & $0,0 \mathrm{C}$ & $0,0 \mathrm{C}$ \\
\hline F tratamento & $47,93^{* *}$ & $501,16^{* *}$ & $30,51^{* *} *$ & $31,62 * *$ & $5.169,34 * *$ \\
\hline $\mathrm{CV}(\%)$ & 28,09 & 4,77 & 22,34 & 21,97 & 1,47 \\
\hline DMS & 14,5 & 5,1 & 23,0 & 22,9 & 1,7 \\
\hline
\end{tabular}

** significativo pelo teste $\mathrm{F}$ a $5 \%$ de probabilidade. Médias seguidas de mesma letra na colu na não diferem estatisticamente entre si pelo teste $t$ a $5 \%$ de probabilidade. DAA - dias após a aplicação do herbicida.

Tabela 4 - Densidade e biomassa das plantas de Sida rhombifolia nos diferentes posicionamentos do herbicida metribuzin associado à palha de milheto

\begin{tabular}{|c|c|c|c|c|c|c|}
\hline \multirow{2}{*}{ Tratamento } & \multicolumn{5}{|c|}{ Densidade de plantas por vaso } & \multirow{2}{*}{$\begin{array}{c}\text { Massa } \\
\text { seca } \\
(\mathrm{g})\end{array}$} \\
\hline & 7 DAA & $14 \mathrm{DAA}$ & $21 \mathrm{DAA}$ & $28 \mathrm{DAA}$ & 35 DAA & \\
\hline 1 - metribuzin sobre a palha - chuva $(30 \mathrm{~mm})$ & $39,5 \mathrm{C}$ & $0,7 \mathrm{D}$ & $0,5 \mathrm{~B}$ & $0,0 \mathrm{~B}$ & $0,0 \mathrm{C}$ & $0,00 \mathrm{C}$ \\
\hline 2 - chuva $(30 \mathrm{~mm}$ ) sobre o solo - metribuzin sobre a palha seca & $69,5 \mathrm{AB}$ & $3,5 \mathrm{D}$ & $0,5 \mathrm{~B}$ & $0,5 \mathrm{~B}$ & $0,5 \mathrm{C}$ & $0,02 \mathrm{C}$ \\
\hline 3 - chuva $(30 \mathrm{~mm})$ - metribuzin sobre a palha seca em pós-emergência & $0,0 \mathrm{E}$ & $48,2 \mathrm{C}$ & $0,0 \mathrm{~B}$ & $0,0 \mathrm{~B}$ & $0,0 \mathrm{C}$ & $0,00 \mathrm{C}$ \\
\hline 4 - chuva $(30 \mathrm{~mm})$ no solo - metribuzin sob a palha seca & $64,5 \mathrm{AB}$ & $2,5 \mathrm{D}$ & $0,2 \mathrm{~B}$ & $0,0 \mathrm{~B}$ & $0,0 \mathrm{C}$ & $0,00 \mathrm{C}$ \\
\hline 5 - metribuzin sobre a palha seca - chuva $(2.5 \mathrm{~mm})$ & $13,0 \mathrm{DE}$ & $14,0 \mathrm{D}$ & $2,2 \mathrm{~B}$ & $0,0 \mathrm{~B}$ & $0,0 \mathrm{C}$ & $0,00 \mathrm{C}$ \\
\hline 6 - metribuzin sobre a palha seca - sem ocorrência de chuvas & $15,2 \mathrm{DE}$ & $0,5 \mathrm{D}$ & $0,5 \mathrm{~B}$ & $0,0 \mathrm{~B}$ & $0,0 \mathrm{C}$ & $0,00 \mathrm{C}$ \\
\hline 7 - metribuzin sem palha - sem ocorrência de chuvas & $32,5 \mathrm{CD}$ & $14,0 \mathrm{D}$ & $4,0 \mathrm{~B}$ & $1,0 \mathrm{~B}$ & $2,5 \mathrm{C}$ & $0,00 \mathrm{C}$ \\
\hline 8 - chuva $(30 \mathrm{~mm})$ sobre a palha - metribuzin & $50,7 \mathrm{BC}$ & $7,7 \mathrm{D}$ & $2,0 \mathrm{~B}$ & $2,5 \mathrm{~B}$ & $1,0 \mathrm{C}$ & $0,07 \mathrm{C}$ \\
\hline 9 - Testemunha com palha & $73,7 \mathrm{AB}$ & $93,5 \mathrm{~B}$ & $71,5 \mathrm{~A}$ & $78,5 \mathrm{~A}$ & $78,5 \mathrm{~A}$ & $3,25 \mathrm{~B}$ \\
\hline 10 - Testemunha sem palha & $82,0 \mathrm{~A}$ & $112,0 \mathrm{~A}$ & $82,7 \mathrm{~A}$ & $82,2 \mathrm{~A}$ & $85,2 \mathrm{~A}$ & $4,75 \mathrm{~A}$ \\
\hline F tratamento & $12,36 * *$ & $42,98^{* * *}$ & $49,77^{* *} *$ & $146,67 * *$ & $98,35^{* *}$ & $47,51 * *$ \\
\hline $\mathrm{CV}(\%)$ & 36,81 & 42,36 & 55,45 & 33,82 & 38,62 & 52,50 \\
\hline DMS & 23,4 & 18,1 & 13,1 & 8,2 & 9,9 & 0,72 \\
\hline
\end{tabular}

** significativo pelo teste $\mathrm{F}$ a $5 \%$ de probabilidade. Médias seguidas de mesma letra na coluna não diferem estatisticamente entre si pelo teste $t$ a 5\% de probabilidade. DAA - dias após a aplicação do herbicida.

Tabela 5 - Porcentagem de controle das plantas de Ipomoea grandifolia nos diferentes períodos de permanência do metribuzin na palha antes da ocorrência da primeira chuva

\begin{tabular}{|l|c|c|c|c|c|}
\hline \multirow{2}{*}{ Período } & \multicolumn{5}{|c|}{ Porcentagem de controle } \\
\cline { 2 - 6 } & $7 \mathrm{DAA}$ & $14 \mathrm{DAA}$ & $21 \mathrm{DAA}$ & $28 \mathrm{DAA}$ & $35 \mathrm{DAA}$ \\
\hline 0 DIAS & $60,0 \mathrm{~A}$ & $100,0 \mathrm{~A}$ & $99,5 \mathrm{~A}$ & $100,0 \mathrm{~A}$ & $100,0 \mathrm{~A}$ \\
\hline 7 DIAS & $45,0 \mathrm{~A}$ & $96,7 \mathrm{~A}$ & $97,0 \mathrm{~A}$ & $97,5 \mathrm{~A}$ & $96,0 \mathrm{AB}$ \\
\hline 14 DIAS & $31,2 \mathrm{AB}$ & $74,5 \mathrm{AB}$ & $91,5 \mathrm{~A}$ & $85,5 \mathrm{~A}$ & $37,2 \mathrm{~B}$ \\
\hline 21 DIAS & $0,0 \mathrm{~B}$ & $62,2 \mathrm{~B}$ & $58,7 \mathrm{~B}$ & $51,2 \mathrm{~B}$ & $37,7 \mathrm{C}$ \\
\hline 28 DIAS & $38,7 \mathrm{~A}$ & $27,5 \mathrm{C}$ & $26,2 \mathrm{C}$ & $33,7 \mathrm{~B}$ & $32,5 \mathrm{C}$ \\
\hline Testemunha & $0,0 \mathrm{~B}$ & $0,0 \mathrm{C}$ & $0,0 \mathrm{D}$ & $0,0 \mathrm{C}$ & $0,0 \mathrm{D}$ \\
\hline F tratamento & $5,30^{* *}$ & $13,77^{* *}$ & $55,02^{* *}$ & $23,60^{* *}$ & $43,50^{* *}$ \\
\hline CV $(\%)$ & 72,9 & 35,4 & 18,0 & 26,9 & 21,7 \\
\hline DMS & 31,6 & 31,7 & 16,6 & 24,5 & 18,3 \\
\hline
\end{tabular}

** significativo pelo teste $\mathrm{F}$ a $5 \%$ de probabilidade. Médias seguidas de mesma letra na coluna não diferem estati sticamente entre si pelo teste $t$ a $5 \%$ de probabilidade. DAA - dias após a aplicação do herbicida. 
Efeito da cobertura morta de milheto (Pennisetum americanum) ...

Tabela 6 - Densidade e biomassa seca das plantas de Ipomoea grandifolia nos diferentes períodos de permanência do metribuzin na palha antes da ocorrência da primeira chuva

\begin{tabular}{|c|c|c|c|c|c|c|}
\hline \multirow{2}{*}{ Período } & \multicolumn{5}{|c|}{ Densidade de plantas por vaso } & \multirow{2}{*}{$\begin{array}{c}\text { Massa seca } \\
(\mathrm{g})\end{array}$} \\
\hline & $7 \mathrm{DAA}$ & $14 \mathrm{DAA}$ & $21 \mathrm{DAA}$ & $28 \mathrm{DAA}$ & $35 \mathrm{DAA}$ & \\
\hline 0 DIAS & $17,5 \mathrm{~A}$ & $0,0 \mathrm{C}$ & $0,5 \mathrm{D}$ & $0,0 \mathrm{C}$ & $0,5 \mathrm{C}$ & $0,0 \mathrm{C}$ \\
\hline 7 DIAS & $16,3 \mathrm{~A}$ & $1,8 \mathrm{BC}$ & $0,8 \mathrm{D}$ & $0,0 \mathrm{C}$ & $0,8 \mathrm{C}$ & $0,5 \mathrm{C}$ \\
\hline 14 DIAS & $20,0 \mathrm{~A}$ & $9,3 \mathrm{~B}$ & $2,5 \mathrm{D}$ & $5,0 \mathrm{BC}$ & $4,0 \mathrm{BC}$ & $1,5 \mathrm{C}$ \\
\hline 21 DIAS & $26,3 \mathrm{~A}$ & $3,5 \mathrm{BC}$ & $9,3 \mathrm{C}$ & $9,8 \mathrm{~B}$ & $13,0 \mathrm{~B}$ & $5,0 \mathrm{~B}$ \\
\hline 28 DIAS & $14,5 \mathrm{~A}$ & $28,0 \mathrm{~A}$ & $20,0 \mathrm{~B}$ & $31,0 \mathrm{~A}$ & $30,0 \mathrm{~A}$ & $5,1 \mathrm{~B}$ \\
\hline Testemunha & $22,5 \mathrm{~A}$ & $28,8 \mathrm{~A}$ & $26,3 \mathrm{~A}$ & $26,5 \mathrm{~A}$ & $29,5 \mathrm{~A}$ & $9,8 \mathrm{~A}$ \\
\hline F tratamento & $1,01^{\text {ns }}$ & $19,61^{\text {ns }}$ & $27,87^{* * *}$ & $19,93^{* *}$ & $15,95 * *$ & $13,60^{* *}$ \\
\hline CV $(\%)$ & 44,26 & 50,03 & 41,91 & 50,28 & 53,93 & 55,46 \\
\hline D.M.S & 12,82 & 8,82 & 6,15 & 8,99 & 10,31 & 2,98 \\
\hline
\end{tabular}

** significativo pelo teste $\mathrm{F}$ a $5 \%$ de probabilidade. Médias seguidas de mesma letra na coluna não diferem estati sticamente entre si pelo teste $t$ a $5 \%$ de probabilidade. DAA - dias após a aplicação do herbicida.

Tabela 7 - Porcentagem de controle das plantas daninhas de Sida rhombifolia nos diferentes períodos de permanência do metribuzin na palha antes da ocorrência da primeira chuva. Botucatu-SP, 2006

\begin{tabular}{|c|c|c|c|c|c|}
\hline \multirow{2}{*}{ Período } & \multicolumn{5}{|c|}{ Porcentagem de controle } \\
\hline & $7 \mathrm{DAA}$ & 14 DAA & $21 \mathrm{DAA}$ & $28 \mathrm{DAA}$ & 35 DAA \\
\hline 0 DIAS & $12,5 \mathrm{C}$ & $99,0 \mathrm{~A}$ & $99,0 \mathrm{~A}$ & $99,7 \mathrm{~A}$ & $100,0 \mathrm{~A}$ \\
\hline 7 DIAS & $87,5 \mathrm{~A}$ & $96,5 \mathrm{~A}$ & $99,5 \mathrm{~A}$ & $72,5 \mathrm{AB}$ & $97,7 \mathrm{AB}$ \\
\hline 14 DIAS & $73,7 \mathrm{~A}$ & $88,7 \mathrm{~A}$ & $97,2 \mathrm{~A}$ & $94,2 \mathrm{AB}$ & $92,2 \mathrm{AB}$ \\
\hline 21 DIAS & $36,2 \mathrm{~B}$ & $91,75 \mathrm{~A}$ & $87,0 \mathrm{~B}$ & $93,5 \mathrm{AB}$ & $89,2 \mathrm{~B}$ \\
\hline 28 DIAS & $38,7 \mathrm{~B}$ & $58,75 \mathrm{~B}$ & $46,2 \mathrm{C}$ & 66,2 B & $66,2 \mathrm{C}$ \\
\hline Testemunha & $0,0 \mathrm{C}$ & $0,0 \mathrm{C}$ & $0,0 \mathrm{D}$ & $0,0 \mathrm{C}$ & $0,0 \mathrm{E}$ \\
\hline F tratamento & $34,40^{* * *}$ & $90,00^{* * *}$ & $247,60 * *$ & $13,40^{* * *}$ & $129,70^{* * *}$ \\
\hline CV $(\%)$ & 27,9 & 11,2 & 7,2 & 28,6 & 9,1 \\
\hline DMS & 17,2 & 12,0 & 7,6 & 30,2 & 10,0 \\
\hline
\end{tabular}

** significativo pelo teste $\mathrm{F}$ a $5 \%$ de probabilidade. Médias seguidas de mesma letra na coluna não diferem estatisticamente entre si pelo teste $t$ a $5 \%$ de probabilidade. DAA - dias após a aplicação do herbicida.

Tabela 8 - Densidade e biomassa seca de plantas de Sida rhombifolia nos diferentes períodos de permanência do metribuzin na palha antes da ocorrência da primeira chuva

\begin{tabular}{|c|c|c|c|c|c|c|}
\hline \multirow{2}{*}{ Período } & \multicolumn{5}{|c|}{ Densidade de plantas por vaso } & \multirow{2}{*}{$\begin{array}{c}\text { Massa seca } \\
(\mathrm{g})\end{array}$} \\
\hline & 7 DAA & 14 DAA & $21 \mathrm{DAA}$ & $28 \mathrm{DAA}$ & 35 DAA & \\
\hline 0 DIAS & $54,3 \mathrm{AB}$ & $1,0 \mathrm{C}$ & $1,3 \mathrm{C}$ & $0,0 \mathrm{C}$ & $0,0 \mathrm{C}$ & $0,0 \mathrm{D}$ \\
\hline 7 DIAS & $12,8 \mathrm{C}$ & $2,8 \mathrm{C}$ & $0,8 \mathrm{C}$ & $0,0 \mathrm{C}$ & $1,5 \mathrm{C}$ & $0,3 \mathrm{CD}$ \\
\hline 14 DIAS & $24,3 \mathrm{C}$ & $13,8 \mathrm{C}$ & $1,0 \mathrm{C}$ & $4,5 \mathrm{C}$ & $6,3 \mathrm{C}$ & $0,0 \mathrm{D}$ \\
\hline 21 DIAS & $30,5 \mathrm{BC}$ & $6,0 \mathrm{C}$ & $4,3 \mathrm{C}$ & $\begin{array}{r}4,8 \mathrm{C} \\
.\end{array}$ & $3,8 \mathrm{C}$ & $3,0 B C$ \\
\hline 28 DIAS & $30,0 \mathrm{C}$ & $51,3 \mathrm{~B}$ & $46,8 \mathrm{~B}$ & $64,0 \mathrm{~B}$ & $58,0 \mathrm{~B}$ & $5,0 \mathrm{AB}$ \\
\hline Testemunha & $63,0 \mathrm{~A}$ & $110,3 \mathrm{~A}$ & $113,8 \mathrm{~A}$ & $116,5 \mathrm{~A}$ & $115,3 \mathrm{~A}$ & $7,8 \mathrm{~A}$ \\
\hline $\mathrm{F}$ tratamento & $5,63 * *$ & $18,02 * *$ & $32,40 * *$ & $30,25 * *$ & $40,21 * *$ & $10,36^{* *}$ \\
\hline $\mathrm{CV}(\%)$ & 44,77 & 65,94 & 57,46 & 55,64 & 48,18 & 54,54 \\
\hline D.M.S & 23,80 & 30,20 & 23,86 & 26,14 & 22,16 & 2,96 \\
\hline
\end{tabular}

** significativo pelo teste $\mathrm{F}$ a $5 \%$ de probabilidade. Médias seguidas de mesma letra na coluna não diferem estatisticamente entre si pelo teste $t$ a $5 \%$ de probabilidade. DAA - dias após a aplicação do herbicida.

De maneira geral, observou-se que o herbicida metribuzin promoveu excelente controle das duas espécies estudadas, nos diferentes posicionamentos testados, exceto para
I. grandifolia na condição de aplicação em palha úmida, seguido de período seco. Demonstrouse, ainda, a ocorrência de absorção pelo contato direto das plantas daninhas com a palha (sem 
ocorrência de chuva após a aplicação). Pode-se concluir também que, quanto maior o tempo de permanência do herbicida na palha antes da ocorrência da primeira chuva, menor sua eficácia de controle das plantas daninhas estudadas.

\section{LITERATURA CITADA}

AZANIA, A. A. P. M. et al. Interferência da palha de canade-açúcar (Saccharum spp.) na emergência de espécies de plantas daninhas da família Convulvulaceae. Planta Daninha, v. 20, n. 2, p. 207-212, 2000.

BANKS, P. A.; ROBINSON, E. L. The influence of straw mulch on the soil reception and persistence of metribuzin. Weed sci., v. 30, n. 2, p. 164-168, 1982.

CORREIA, N. M.; DURIGAN, J. C. Emergência de plantas daninhas em solo coberto com palha de cana-de-açúcar.

Planta Daninha, v. 22, n. 1, p. 11-18, 1994.

LORENZI, H. Consideração sobre plantas daninhas no plantio direto. In: TORRADO, P. V.; ALOISI, R. R. Plantio Direto Brasil. Campinas: CARGILL, 1984. p. 13-46.

LORENZI, H. J. Efeito da palha de cana no controle de plantas daninhas. In: CONGRESSO BRASILEIRO DE HERBICIDAS E PLANTAS DANINHAS, 19., 1993, Londrina. Resumos... Londrina: SBHED, 1993. p. 28-29.

MACIEL, C. D. G. Simulação do caminhamento de herbicidas em diferentes tipos e quantidades de palhadas utilizadas no sistema de plantio direto. 2001. $89 \mathrm{f}$. Tese (Mestrado em Agronomia) - Universidade Estadual Paulista, Botucatu, 2001.
MACIEL, C. D. G.; VELINI, E. D. Simulação do caminhamento da água da chuva e herbicidas em palhadas utilizadas em sistema de plantio direto. Planta Daninha, v. 23, n. 3, p. 471-482, 2005.

MARTINS, D. et al. Emergência em campo de dicotiledôneas infestantes em solo coberto com palha de cana-de-açúcar. Planta Daninha, v. 17, n. 1, p. 151-161, 1999.

PEREZ, J. F. et al. Determination of the herbicide metribuzin and its major conversion products in soil by micellar electrokinetic chromatography. J. Chromatog., v. 1102, p. 280-286, 2006.

RODRIGUES, B. N. et al. Influência da cobertura morta no comportamento do herbicida trifluralin. Planta Daninha, v. 16, n. 2, p. 163-173, 1998.

ROSSI, C. V. S. Dinâmica e eficácia no controle de plantas daninhas pelo herbicida metribuzin aplicado sobre palha de cana-de-açúcar. 2004. 95 f. Dissertação (Mestrado em Agronomia/Proteção de Plantas) - Universidade Estadual Paulista, Botucatu, 2004.

SAVAGE, K.E. Metribuzin persistence on the soil surface. Weed Sci., v. 33, p. 288, 1980.

TOFOLI, G. R. Deposição e lixiviação do herbicida tebuthiuron em palha de cana-de-açúcar. 2004. 63 f. Tese (Doutorado em Agronomia/Proteção de Plantas) Universidade Estadual Paulista, Botucatu, 2004.

VELINI, E.D.; NEGRISOLI, E. Controle de plantas daninhas em canacrua. In: CONGRESSO BRASILEIRO DA CIÊNCIA DAS PLANTAS DANINHAS, 22., 2000, Foz do Iguaçu. Palestras... Foz do Iguaçu: Sociedade Brasileira da Ciência das Plantas Daninhas, 2000. p. 148-164. 